\title{
PARP inhibition in castration-resistant prostate cancer
}
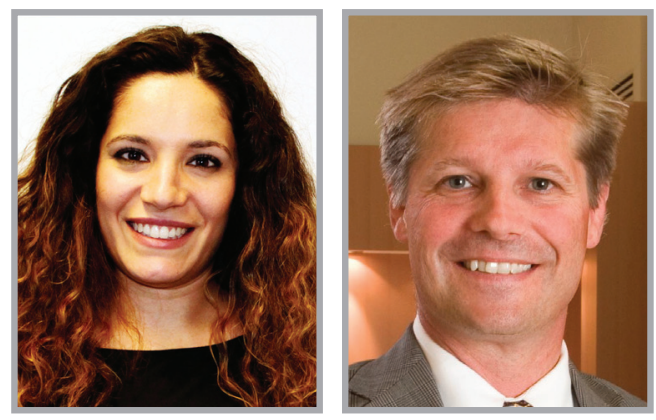

\author{
“Poly(ADP-ribose) polymerase \\ inhibitors could represent a \\ new therapeutic option for a \\ large percentage of patients \\ with castration-resistant \\ prostate cancer harboring DNA \\ repair gene mutations."
}

Lucia Nappi \& Martin E Gleave ${ }^{*, 1}$

First draft submitted: 15 December 2015; Accepted for publication: 4 January 2016; Published online: 21 January 2016

Prostate cancer $(\mathrm{PCa})$ is one of the most common adult malignancies, with 220,000 diagnosed men every year in USA [1]. Androgen-deprivation therapy is the first line of therapy in patients with metastatic disease and despite its great efficacy nearly all patients develop castration-resistant prostate cancer (CRPC) and eventually die from it [2]. Although AR still plays a fundamental role in the castration-resistant disease and can be effectively targeted by more potent androgen receptor pathway inhibitor, like enzalutamide and abiraterone, accumulating evidence support roles for alternative pathways such as CRPC drivers $[3,4]$. DNA repair defects have been identified in many sporadic and hereditary tumors. BRCA1 and BRCA2 are the best characterized DNA repair genes associated with cancer development. These proteins have a crucial role in the regulation of homologous recombination (HR) repair, an accurate DNA double-strand break (DSB) repair process. In the absence of BRCAs (and other HR proteins), DNA DSBs increase which induce the accumulation of DNA mutations and thereby promotes tumorigenesis [5]. Although BRCA dysfunction promotes an oncogenic advantage, it also renders cancer cells reliant on alternative DNA repair pathways, such as base excision repair (BER). Poly(ADPribose) polymerase are a family of enzymes that catalyze $\mathrm{NAD}^{+}$-dependent ADPribosylation of DNA. PARP1 and PARP2 are the best characterized members of the PARP family and they share similar structure and functions. PARP1 has been implicated in several DNA repair mechanisms, including DNA single-strand breaks (SSB) repair through the BER pathway. PARP1 recognizes DNA SSB and orchestrates the recruitment and assembly of a DNA repair complex [6]. Therefore, PARP inhibition induces unrepaired SSB accumulation that is converted into DSBs at fork replication. Cells with deficient HR repair system (i.e., for $B R C A 1 / 2$ mutations) treated with PARP inhibitors are overcome by DNA DSBs which lead to further chromosomal instability, cell cycle arrest and apoptosis. Thus, HR dysfunction sensitizes

\section{KEYWORDS}

- castration-resistant prostate cancer $\bullet$ DNA repair gene mutations - gene sequencing $\bullet$ PARP inhibitors

- targeted therapy 
"The identification of genetic biomarkers for olaparib response leads the way to a new concept of clinical trial and to the development of new techniques to improve the costs and the availability of

biological data that can drive the choice of the best therapy..." cells to PARP inhibition. This synthetic lethality represents the rationale for the use of PARP inhibitors in cancers harboring HR DNA repair alterations [7]. PARP inhibitors (PARPi) are a class of drugs designed to compete with $\mathrm{NAD}^{+}$ for the substrate binding site of both PARP1 and PARP2, acting as catalytic inhibitors. However, $\mathrm{NAD}^{+}$catalytic competition is only one of the mechanisms of action of PARPi in DNA repair pathway modulation. As recently discovered, PARPi are also able to trap PARP, preventing its activation for DNA repair and causing replication and transcription fork blockade with subsequent DNA breakage. The effect on PARP trapping is not correlated to PARP catalytic inhibition and differs among the different PARPi [8]. Aside from its role in DNA damage response, PARP has been implicated in AR transcription regulation, AR target gene expression and tumor proliferation in PCa [9]. PARPi have demonstrated antitumor activity in preclinical models of TMPRSS2-ERG rearranged prostate cancer [10].

\section{PARP inhibition in germline-mutated PCa}

Although PARPi efficacy has been established for patients with breast and ovarian cancer harboring germline BRCA1/2 mutations [11], preliminary data also showed the activity of these drugs in patients with germline BRCA1/2-mutated PCa. These rare tumors are particularly frequent in young patients ( $<65$ years), with $B R C A 2$ more prevalent than BRCA1 (1.4 and $0.44 \%$ of all $\mathrm{PCa}$, respectively). Germline mutations carriers have higher Gleason score, lower overall survival (OS) and cancer-specific survival, higher advanced stages and globally a worse prognosis compared with noncarriers patients [12]. In a Phase II clinical trial, Kaufman et al. [13] reported $50 \%$ response rate, $25 \%$ stable disease and overall median duration response of 327 days in eight patients with germline BRCA1/2 mutations treated with olaparib. Median progression-free survival was 7.2 months with $62.5 \%$ of patients still progression-free at 6 months. Moreover, the OS was 18.4 months and the proportion of patients still alive at 12 months was $50 \%$. Interestingly, this study also showed that exposure to cisplatin is a marker of poor response to PARPi in patients with CRPC. Responses to PARPi were reported in four patients with advanced $\mathrm{PCa}$ and germline BRCA2 mutations treated on three Phase I studies [14]. Interestingly, all four patients presented PTEN deletions and $E R G$ rearrangements. This higher rate, compared with the expected $50-60 \%$ rate in $\mathrm{PCa}$, suggests that HR defects could play a role in the alterations of these genes.

\section{PARP inhibition in somatic-mutated PCa}

While only a minority of PCa patients carry germline mutations, many sporadic CRPC harbor epigenetic and genetic disruption of genes that are crucial for the homologous recombination pathway, such as BRCA1, BRCA2, FANC, ATM, CHEK2, MRE11A and RAD51 [15]. Some of these aberrations have been associated with sensitivity to platinum and PARPi [16], suggesting a synthetic lethal interaction between platinum/PARP inhibitors and these sporadic DNA repair gene defects. In a Phase I dose-escalation clinical trial, Sandhu et al. treated 21 patients affected by sporadic CRPC with niraparib, a new PARP inhibitor, at the dose of 290-300 mg. The results reported stabilization of CRPC disease in 43\% of patients with a median duration of response of 254 days. In total, $30 \%$ of patients had a decrease of circulating tumor cells (CTCs) and one of the 21 patients enrolled had $>50 \%$ PSA reduction. They did not observe correlation between $E R G$ rearrangements/loss of PTEN expression and treatment response [17]. This study did not analyze the genetic alteration in HR genes in patients with CRPC. Recent genetic studies have showed somatic mutations of DNA HR repair system in more than $20 \%$ of the patients with CRPC. Interestingly, the genes identified are involved in different steps and mechanisms of HR machinery [15]. In an extensive genome analysis, Robinson et al. compared genetic sequencing data of castration sensitive and CRPC. BRCA2 was the most frequent mutations occurring in $12.7 \%$ of cases. Interestingly, the analysis of other DNA repair genes showed an overall DNA repair gene aberrations in $22.7 \%$ of patients with $A T M$ and BRCA1 the most frequent alterations (in $19.3 \%$ of patients) and few patients $(3.4 \%$ in total) reporting CDK12, FANCA, RAD51B and RAD51C mutations [15].

\section{DNA repair gene abnormalities as response biomarkers to PARPi in PCa}

The association between somatic DNA repair genes mutations and the response to PARP inhibitor olaparib has been recently investigated by Mateo et al. in a multicenter Phase II clinical trial [18]. Overall, 50 patients with mCRPC after progression to one to two cycles of chemotherapy were enrolled to receive olaparib at the 
dose of $400 \mathrm{mg}$ twice a day. Patients with a previous exposure to platinum were excluded. For the biomarkers study, whole-exome sequencing and transcriptome analysis were performed on DNA from fresh-frozen tumor biopsy tissue obtained before treatment while the germline whole-exome sequencing was performed on DNA from saliva samples. Patients were divided in two populations, biomarkers positive or biomarkers negative, according to the identification of homozygous deletion or deleterious mutations in genes reported to be involved either in DNA damage repair or sensitivity to PARP inhibition. Moreover, PTEN and ERG protein expression was determined in the tumor samples to assess the correlation between the response to olaparib and PTEN/ERG status.

The enrolled patients were heavily pretreated: all the patients had received docetaxel and 58\% had been treated with cabazitaxel, 96 and $28 \%$ of the patients had been treated with abiraterone and enzalutamide, respectively. The overall response rate was $33 \%$ and the median overall survival was 10.1 months. Most interestingly, $88 \%$ of biomarker-positive patients had a response to olaparib. These patients also had prolonged progression-free survival and OS compared with biomarker negative population (9.8 vs 2.7 months and 13.8 vs 7.5 months, respectively). BRCA2 was the most frequent DNA repair gene alteration and all the patients carrying it responded to olaparib. Four of the five patients with ATM abnormalities and all the patients harboring less common DNA repair genes mutations (in PALB2, FANCA and $H D A C 2$ ) had a response to olaparib. One patient carrying $M L H 3$ loss and one with ATM mutation did not respond to olaparib. Conversely only two $(6 \%)$ of patients defined as biomarker negative responded to the treatment. There was no correlation between olaparib response and PTEN/ERG mutational status. This trial demonstrated the activity of PARP inhibition as therapeutic agent in patients with $\mathrm{mCRPC}$ and somatic mutations of HR DNA genes, confirming the synthetic lethality between PARPi and DNA HR also in sporadic tumors. Although the study clearly showed that genetic biomarkers could be used to select the patients with mCRPC for specific targeted drugs, gene sequencing is not available as standard test in the clinical practice. However, the creation of specific probes to identify the specific genes alterations can significantly simplify the process of genes analysis and the use of CTC and/or on tumor cell free DNA as source of tumor DNA can avoid invasive procedures to obtain tumor samples [19].

PARPi could represent a new therapeutic option for a large percentage of patients with CRPC harboring DNA repair gene mutations. Almost 30\% of patients with CRPC have these alterations and they have high possibility to respond to the treatment. Moreover, the presence of these mutations could also sensitize these tumor cells to platinum and other chemotherapy agents, as demonstrated for various solid tumors [20].

\section{Conclusion}

In summary, this study promotes the use of PARP inhibitors as a new therapeutic strategy for CRPC tailored to the genomic features of the tumor. The identification of genetic biomarkers for olaparib response leads the way to a new concept of clinical trial and to the development of new techniques to improve the costs and the availability of biological data that can drive the choice of the best therapy, especially in CRPC that is a high genetic heterogeneous disease.

\section{Financial \& competing interests disclosure}

The authors have no relevant affiliations or financial involvement with any organization or entity with a financial interest in or financial conflict with the subject matter or materials discussed in the manuscript. This includes employment, consultancies, honoraria, stock ownership or options, expert testimony, grants or patents received or pending, or royalties.

No writing assistance was utilized in the production of this manuscript.

\section{References}

1 American Cancer Society. Cancer Facts \& Figures 2015.

www.cancer.org

2 Sridhar SS, Freedland SJ, Gleave ME et al. Castration-resistant prostate cancer: from new pathophysiology to new treatment. Eur. Urol. 65(2), 289-299 (2014).
Beltran H, Eng K, Mosquera JM et al. Wholeexome sequencing of metastatic cancer and biomarkers of treatment response. JAMA Oncol. 1(4), 466-474 (2015).

4 Wyatt AW, Gleave ME. Targeting the adaptive molecular landscape of castrationresistant prostate cancer. EMBO Mol. Med. 7(7), 878-894 (2015).
5 Underhill C, Toulmonde M, Bonnefoi H. A review of PARP inhibitors: from bench to bedside. Ann. Oncol. 22(2), 268-279 (2011).

6 Do K, Chen AP. Molecular pathways: targeting PARP in cancer treatment. Clin. Cancer Res. 19(5), 977-984 (2013).

7 Ashworth A. A synthetic lethal therapeutic approach: poly(ADP) ribose polymerase 
inhibitors for the treatment of cancers deficient in DNA double-strand break repair J. Clin. Oncol. 26(22), 3785-3790 (2008).

8 Murai J, Huang SY, Das BB et al. Trapping of PARP1 and PARP2 by clinical PARP inhibitors. Cancer Res. 72(21), 5588-5599 (2012).

9 Brenner JC, Ateeq B, Li Y et al. Mechanistic rationale for inhibition of poly(ADP-ribose) polymerase in ETS gene fusion-positive prostate cancer. Cancer Cell 19(5), 664-678 (2011).

10 Chatterjee P, Choudhary GS, Sharma A et al. PARP inhibition sensitizes to low dose-rate radiation TMPRSS2-ERG fusion geneexpressing and PTEN-deficient prostate cancer cells. PLoS ONE 8(4), e60408 (2013).

11 Fong PC, Boss DS, Yap TA et al. Inhibition of poly(ADP-ribose) polymerase in tumors from BRCA mutation carriers. N. Engl. J. Med. 361(2), 123-134 (2009).
12 Castro E, Goh C, Olmos D et al. Germline $B R C A$ mutations are associated with higher risk of nodal involvement, distant metastasis, and poor survival outcomes in prostate cancer. J. Clin. Oncol. 31(14), 1748-1757 (2013).

13 Kaufman B, Shapira-Frommer R, Schmutzler $\mathrm{RK}$ et al. Olaparib monotherapy in patients with advanced cancer and a germline BRCA1/2 mutation. J. Clin. Oncol. 33(3), 244-250 (2015).

14 Sandhu SK, Omlin A, Hylands L et al. Poly (ADP-ribose) polymerase (PARP) inhibitors for the treatment of advanced germline BRCA2 mutant prostate cancer. Ann. Oncol. 24(5), 1416-1418 (2013)

15 Robinson D, Van Allen EM, Wu YM et al. Integrative clinical genomics of advanced prostate cancer. Cell 161(5), 1215-1228 (2015).

16 Pitroda SP, Pashtan IM, Logan HL et al. DNA repair pathway gene expression score correlates with repair proficiency and tumor sensitivity to chemotherapy. Sci. Transl. Med. 6(229), 229ra242 (2014)

17 Sandhu SK, Schelman WR, Wilding G et al. The poly(ADP-ribose) polymerase inhibitor niraparib (MK4827) in BRCA mutation carriers and patients with sporadic cancer: a Phase 1 dose-escalation trial. Lancet Oncol. 14(9), 882-892 (2013).

18 Mateo J, Carreira S, Sandhu S et al. DNA-repair defects and olaparib in metastatic prostate cancer. N. Engl. J. Med. 373(18), 1697-1708 (2015).

19 Azad AA, Volik SV, Wyatt AW et al. Androgen receptor gene aberrations in circulating cell-free DNA: biomarkers of therapeutic resistance in castration-resistant prostate cancer. Clin. Cancer Res. 21(10), 2315-2324 (2015).

20 Gorodnova TV, Sokolenko AP, Ivantsov AO et al. High response rates to neoadjuvant platinum-based therapy in ovarian cancer patients carrying germ-line $B R C A$ mutation. Cancer Lett. 369 (2), 363-367 (2015). 